\title{
Helminth antigens counteract a rapid high-fat diet-induced decrease in adipose tissue eosinophils
}

\author{
Susan M van den Berg', Andrea D van Dam²,3, Pascal J H Kusters', Linda Beckers', \\ Myrthe den Toom', Saskia van der Velden', Jan Van den Bossche1, Irma van Die ${ }^{4}$, \\ Mariëtte R Boon ${ }^{2,3}$, Patrick C N Rensen 2,3 , Esther Lutgens ${ }^{1,5, *}$ and \\ Menno P J de Winther1,5,*
}

'Department of Medical Biochemistry, Experimental Vascular Biology, Academic Medical Center, University of Amsterdam, Amsterdam, The Netherlands

2Department of Medicine, Division Endocrinology, Leiden University Medical Center, Leiden, The Netherlands ${ }^{3}$ Einthoven Laboratory for Experimental Vascular Medicine, Leiden University Medical Center, Leiden, The Netherlands ${ }^{4}$ Department of Molecular Cell Biology and Immunology, VU University Medical Center, Amsterdam, The Netherlands 5Institute for Cardiovascular Prevention (IPEK), Ludwig Maximilian's University, Munich, Germany

*(E Lutgens and M P J de Winther contributed equally to this work)

Correspondence should be addressed to $M$ P J de Winther Email

m.dewinther@amc.uva.nl

\begin{abstract}
Brown adipose tissue (BAT) activation and white adipose tissue (WAT) beiging can increase energy expenditure and have the potential to reduce obesity and associated diseases. The immune system is a potential target in mediating brown and beige adipocyte activation. Type 2 and anti-inflammatory immune cells contribute to metabolic homeostasis within lean WAT, with a prominent role for eosinophils and interleukin (IL)-4-induced anti-inflammatory macrophages. We determined eosinophil numbers in epididymal WAT (EpAT), subcutaneous WAT (SCAT) and BAT after 1 day, 3 days or 1 week of high-fat diet (HFD) feeding in C57BI/6 mice. One day of HFD resulted in a rapid drop in eosinophil numbers in EpAT and BAT, and after 3 days, in ScAT. In an attempt to restore this HFD-induced drop in adipose tissue eosinophils, we treated 1-week HFD-fed mice with helminth antigens from Schistosoma mansoni or Trichuris suis and evaluated whether the well-known protective metabolic effects of helminth antigens involves BAT activation or beiging. Indeed, antigens of both helminth species induced high numbers of eosinophils in EpAT, but failed to induce beiging. In ScAT, Schistosoma mansoni antigens induced mild eosinophilia, which was accompanied by slightly more beiging. No effects were observed in BAT. To study type 2 responses on brown adipocytes directly, T37i cells were stimulated with IL-4. This increased Ucp1 expression and strongly induced the production of eosinophil chemoattractant CCL11 (+26-fold), revealing that brown adipocytes themselves can attract eosinophils. Our findings indicate that helminth antigen-induced eosinophilia fails to induce profound beiging of white adipocytes.
\end{abstract}

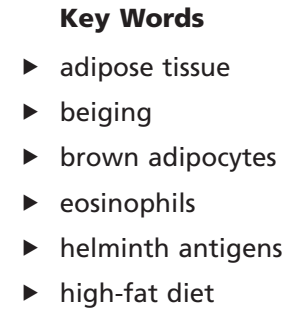

Journal of Molecular Endocrinology (2017) 59, 245-255
○ 2017 Society for Endocrinology Printed in Great Britain 


\section{Introduction}

Brown adipose tissue (BAT) contributes to the control of body temperature by the production of heat in response to cold. Brown adipocytes have numerous mitochondria that contain uncoupling protein 1 (UCP1). Activation of brown adipocytes by noradrenaline results in fatty acid mobilisation and activation of UCP1, thereby uncoupling the respiratory chain from ATP production, resulting in the dissipation of energy into heat (Cannon \& Nedergaard 2004). Rodents as well as newborns from larger organisms, including humans, have interscapular BAT depots. In adult humans, BAT regresses with age but can still be found in supraclavicular and in the neck regions, but also in periaortic, paravertebral and perirenal regions (van Marken Lichtenbelt et al. 2009). BAT activity can be detected by injection of 18F-fluorodeoxyglucose ([18F]FDG) and a positron-emission tomographic and $\mathrm{X}$-ray computed tomography (PET/CT) scanning since the uptake of glucose is high in metabolically active tissues such as BAT (Saito et al. 2009). Interestingly, [18F] FDG uptake by BAT is negatively correlated with BMI, suggestive of decreased BAT activity in obesity (Saito et al. 2009), at least with respect to glucose metabolism. Moreover, activation of BAT by repetitive cold exposure enhances energy expenditure and lowers fat mass (van der Lans et al. 2013, Yoneshiro et al. 2013), underscoring the role of BAT in energy metabolism in humans.

Brown-like or 'beige/brite' adipocytes can also be present within white adipose tissue (WAT) (SanchezGurmaches et al. 2016). These beige adipocytes display similar characteristics as classical brown adipocytes, including a multilocular appearance and Ucp1 expression. They are present at low quantities but their number and activity can be increased by cold or $\beta 3$-adrenergic receptor agonists (Berbée et al. 2015). The inguinal subcutaneous adipose tissue (ScAT) depot has the highest beiging potential after cold exposure (Waldén et al. 2011), although the thermogenic capacity is only $10 \%$ of that of interscapular BAT (Shabalina et al. 2013). Activation of BAT and beige adipocytes increases energy expenditure and therefore has the potential to reduce excessive energy stores in obesity (Yoneshiro \& Saito 2015).

Immune cells contribute to metabolic homeostasis of adipose tissue, and lean WAT and BAT are characterized by an anti-inflammatory immune cell composition (Lumeng et al. 2007). The stromal vascular fraction (SVF) contains high numbers of anti-inflammatory macrophages, eosinophils, CD4+ T helper cells and regulatory $\mathrm{T}$ cells. Anti-inflammatory macrophages are sustained by Th2 cytokines, such as IL-4. In adipose tissue, the majority of IL-4-secreting cells are eosinophils (Wu et al. 2011). In obesity, increased lipid uptake, hypertrophic adipocytes and leakage of fatty acids cause recruitment of pro-inflammatory immune cells. Obese WAT, and BAT to a lower extent, is infiltrated by pro-inflammatory macrophages (Weisberg et al. 2003, Roberts-Toler et al. 2015). Interestingly, eosinophil numbers in WAT inversely correlate with body weight and absence of eosinophils results in increased body weight, impaired glucose tolerance and less cold-induced beige adipogenesis in mice (Wu et al. 2011, Qiu et al. 2014). Anti-inflammatory macrophages have been implicated to induce BAT activation and beiging of WAT, as they release noradrenaline that activates brown and beige adipocytes (Nguyen et al. 2011, Qiu et al. 2014). Furthermore, administration of IL-4 to mice reduces body weight, improves insulin sensitivity and promotes beiging of WAT (Qiu et al. 2014). Thus, BAT activation and WAT beiging are promoted by eosinophils that produce IL-4, which stimulates anti-inflammatory macrophages to release noradrenaline, resulting in the activation and increased expression of UCP1. Decreased BAT activity in obesity and a decrease of both eosinophils and antiinflammatory macrophages in obese WAT suggests that this immunological circuitry is disturbed in an obese state.

Eosinophils are massively increased by helminths, which are parasitic worms that induce a type 2 immune response in their host. Interestingly, such a type 2 response induced by Schistosoma mansoni-soluble egg antigens (SEA) or Nippostrongylus brasiliensis can be protective against metabolic disorders (Wu et al. 2011, Wolfs et al. 2014, Hussaarts et al. 2015). Furthermore, soluble products of the whipworm Trichuris suis (TsSP) also induce a type 2 response and are proven to ameliorate clinical parameters in murine experimental autoimmune encephalomyelitis, a model system for multiple sclerosis (Kuijk et al. 2012, Hoeksema et al. 2016). Whether TsSP affects immune cells in adipose tissue is unknown.

Due to the important role of eosinophils and macrophages in adipose tissue, we first determined how a high-fat diet (HFD) affects these immune cells in BAT, epididymal WAT (EpAT) and ScAT in a short time course study of HFD feeding in mice. The type 2 immune response induced by helminth antigens is metabolically beneficial, and we hypothesized that this phenotype is related to activation of BAT and/or beiging of WAT. We then studied how SEA and TsSP alter the immune composition of these

Published by Bioscientifica Ltd. 
three adipose tissue depots and assessed whether SEA and TsSP administration resulted in BAT activation and beiging of EpAT and ScAT.

\section{Materials and methods}

\section{Mice}

Male wild-type C57Bl/6 mice (Charles River) were fed a HFD (45\% kcal fat, 35\% kcal carbohydrate, 20\% kcal protein, Special Diets Services, Witham, United Kingdom) for 1 day, 3 days or 1 week at the age of 16 weeks. A control group that did not receive HFD was included, resulting in 4 groups of $n=11$. To evaluate the effect of SEA and TsSP, mice were given a HFD for 1 week and

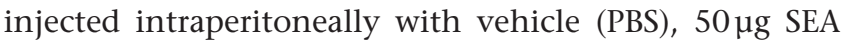
or $50 \mu \mathrm{g}$ TsSP 4 times every 3 days, starting 3 days before the diet ( 3 groups of $n=12$ ). Mice were maintained at a constant temperature of $21^{\circ} \mathrm{C}$, had ad libitum access to food and water and were maintained under a 12-h lightdarkness cycle. Mice were killed using $0.25 \mathrm{mg} / \mathrm{g}$ ketamine and $0.05 \mathrm{mg} / \mathrm{g}$ xylazine. Epididymal adipose tissue (EpAT), ScAT and interscapular BAT were dissected and weighed. All experimental procedures were approved by the Animal Experimentation Ethics Committee of the University of Amsterdam.

\section{SEA and TsSP preparation}

Schistosoma mansoni-SEA were kindly provided by Fred Lewis (Biomedical Research Institute, Rockville, MD, USA) and prepared as described previously (Boros \& Warren 1970). Soluble products of Trichuris suis (TsSP) were kindly provided by Irma van Die and prepared as described (Klaver et al. 2013).

\section{Flow cytometry}

Adipose tissues were collected in PBS, transferred to DMEM-20 mM HEPES (Thermo Fisher Scientific) and minced into small pieces. Digestion was done using $0.25 \mathrm{mg} / \mathrm{mL}$ liberase (Roche) in DMEM-20 mM HEPES for $45 \mathrm{~min}$ at $37^{\circ} \mathrm{C}$. Digested tissue was passed through a $70 \mu \mathrm{m}$ nylon mesh (BD Biosciences, Breda, The Netherlands) and centrifuged at $1250 \mathrm{rpm}$ for $6 \mathrm{~min}$. The adipocyte fraction was removed and the pellet containing the SVF was resuspended in FACS buffer $(0.5 \%$ BSA in PBS). Cell suspensions were incubated with an Fc-receptor blocking antibody to prevent non-specific binding and a biotin-PE-dump antibody mix (BioLegend and eBioscience, San Diego, CA, USA) followed by staining using the antibodies: CD45, CD68 (BioLegend), CD11b, SiglecF, Ly6G (BD Biosciences), CD3, MHCII and F4/80 (eBioscience). Staining was analysed by FACS (LSR Fortessa, BD Biosciences) and FlowJo software (Tree star).

\section{Histology}

Tissues were collected, fixed in $4 \%$ paraformaldehyde and embedded in paraffin. Immunohistochemistry on EpAT and ScAT was performed for UCP1 (SigmaAldrich). The presence of beige adipocytes, defined by a multilocular appearance and positive for UCP1 was scored by M.T., who was blinded for the experimental conditions. When present, the quantity was expressed as ' + ' being a few cells, ' ++ ' a few areas and ' +++ ' when the section was full of beige adipocytes.

\section{Real-time PCR}

Total RNA was extracted using TRIzol (Invitrogen) and reverse transcribed with an iScript cDNA synthesis kit (BioRad). Quantitative PCR was performed using a SYBR green PCR kit and a ViiA7 RT-PCR system (Applied Biosystems). Expression was normalized to the housekeeping genes RplpO, Actb and Ppia. The results are expressed as relative to the control group, which was assigned a value of 1 .

\section{Culture and differentiation of brown adipocytes}

T37i cells were kindly provided by Marc Lombès (Zennaro et al. 1998) and cultured in DMEM-F12 Glutamax, 10\% FCS, penicillin $(100 \mathrm{U} / \mathrm{mL})$ and streptomycin $(100 \mu \mathrm{g} / \mathrm{mL})$ (ThermoFisher Scientific). After growing confluent, cells were differentiated by adding $2 \mathrm{nM}$ triiodothyronine $\left(\mathrm{T}_{3}\right)$ (Sigma-Aldrich) and $112 \mathrm{ng} / \mathrm{mL}$ insulin (Sigma-Aldrich) to the media. After 9 days of differentiation, the cells were stimulated with $20 \mathrm{ng} / \mathrm{mL}$ IL-4 (Peprotech, Rocky Hill, NJ, USA) for $24 \mathrm{~h}$. Supernatant was collected for the quantification of CCL11 (R\&D systems) by ELISA in accordance to the suppliers' protocols.

\section{Statistics}

Results are presented as mean \pm s.E.M. Analyses between more than two groups in the time course experiments were done by a one-way ANOVA with Tukey post-test analysis and the change per group was expressed relative to the control group. Analysis between two groups was done by a Student's $t$ test or a chi-square test. Statistics

Published by Bioscientifica Ltd 
were calculated in GraphPad Prism 5.0 (GraphPad Software, Inc., La Jolla, CA, USA). $P$ values $<0.05$ were considered significant.

\section{Results}

\section{Eosinophils rapidly decrease in adipose tissue upon HFD}

Since adipose tissue eosinophils are involved in beige adipogenesis (Qiu et al. 2014), we were interested in how HFD affects eosinophil numbers in different adipose tissue depots. We determined adipose tissue eosinophil numbers in HFD-fed C57Bl/6 wild-type mice by including very short periods of HFD feeding; 1 day, 3 days and 1 week and compared them to age-matched mice on a chow diet (Fig. 1A). Analysis of adipose tissue immune cells by flow cytometry revealed that already after 1 day of HFD, the numbers of eosinophils declined in EpAT (-35\%) and BAT $(-65 \%)$ and after 3 days in ScAT as well (-36\%; Fig. 1B). We also observed an increase in macrophages after 3 days of HFD in EpAT (+19\%; Fig. 1C). There were no changes in M1- (CD11c) or M2-like (CD301) macrophages within one week of HFD (data not shown). These data show that HFD rapidly alters the immune cell composition of brown and white adipose tissues before the onset of obesity.

To determine how HFD feeding can cause such a rapid decline in eosinophil numbers, we measured the expression of key chemokines that are known to attract eosinophils; Ccl3, Ccl5 and Ccl11. The HFD downregulated the expression of especially $\mathrm{Ccl} 3$ in the WATs (Fig. 2A and B), although Ccl11 was increased in EpAT after 1 week of HFD. Expression of Ccl5 and Ccl11 was decreased in BAT (Fig. 2C). These data show that HFD induces rapid changes in immune cell composition and is accompanied by reduced expression of eosinophilrecruiting chemokines.

\section{IL-4 enhances Ucp1 expression and CCL11 production in brown adipocytes in vitro}

Eosinophils cause beiging of WAT by producing IL-4 that stimulates anti-inflammatory macrophages to secrete noradrenaline (Wu et al. 2011, Lee et al. 2015). To evaluate the direct crosstalk between eosinophils, IL-4 and brown adipocytes, we stimulated a brown adipocyte cell line (T37i) with IL-4 for $24 \mathrm{~h}$. The cell line expresses high levels of the $I l 4 r$ (Ct value around 21, data not shown) and we observed upregulated Ucp1 expression (Fig. 2D), which suggests that IL-4 per se directly activates brown adipocytes, without the necessity of noradrenalinsecreting macrophages. Interestingly, we found that stimulation of the brown adipocytes with IL-4 massively increased the expression and secretion of CCL11 (Fig. 2E and F), while IL-4 had limited effect on Ccl5 (2-fold increase) and no effect on $\mathrm{Ccl3}$ (data not shown). Altogether, these data suggest a mechanism in which HFD feeding suppresses the activation of BAT and possibly the
A

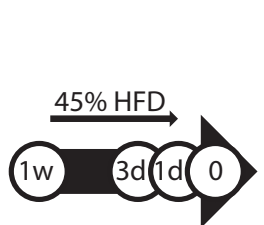

4 groups of mice

- No HFD

-1 day

-3 days

-1 week
B

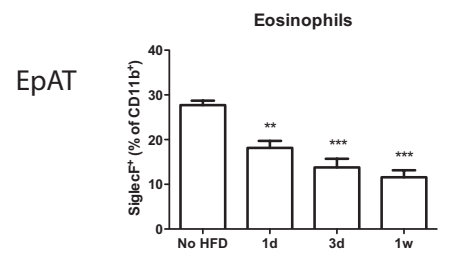

Eosinophils

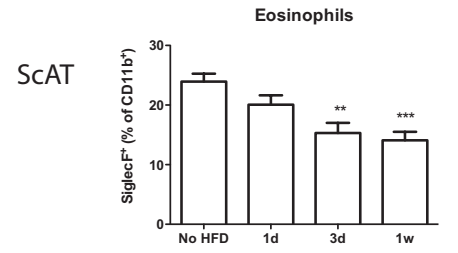

Eosinophils

BAT
C

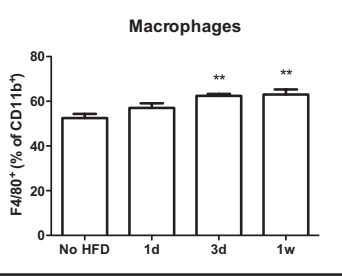

Macrophages

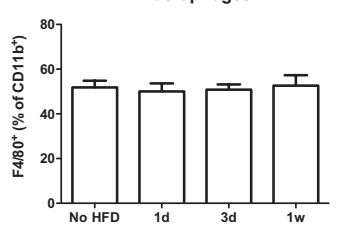

Macrophages

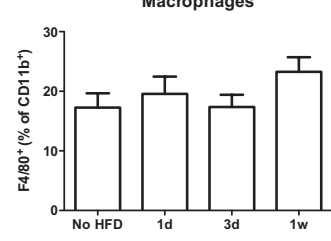

\section{Figure 1}

High-fat diet feeding rapidly decreases the number of adipose tissue eosinophils. (A) Schematic study outline. (B) Flow cytometry analysis of eosinophils (SiglecF+ leukocytes) and (C) macrophages ( $\mathrm{F} 4 / 80^{+}$leukocytes) in the stromal vascular fraction of EPAT, ScAT and BAT after 1 day, 3 days or 1 week of HFD. $n=11$, ${ }^{*} P<0.05, * * P<0.01, * * * P<0.001$ (1-way ANOVA with Tukey post-test analysis and the statistical change per group expressed relative to the No HFD group). 
A

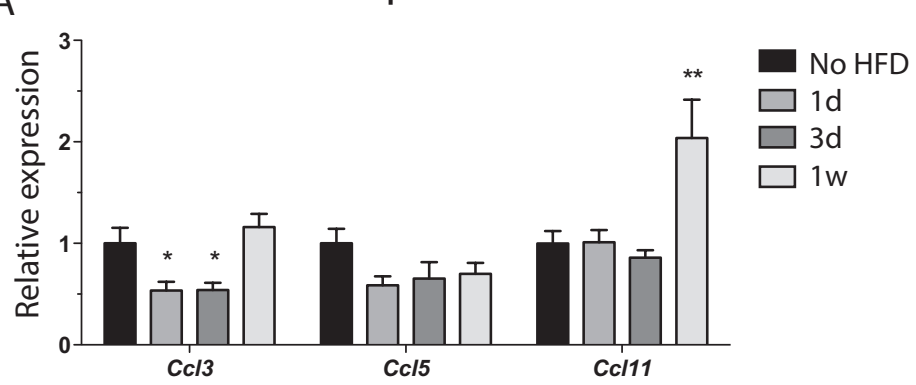

B

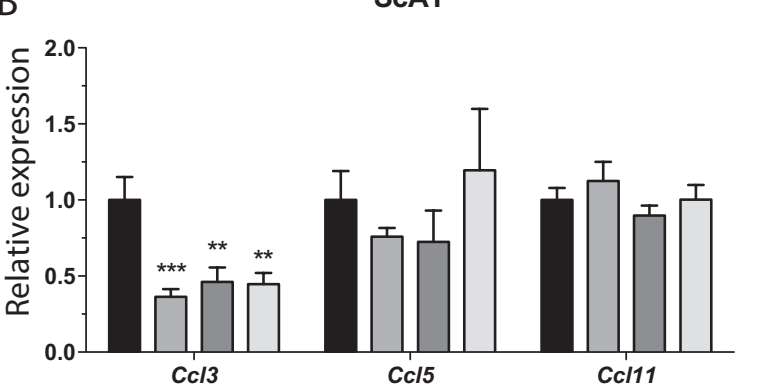

C

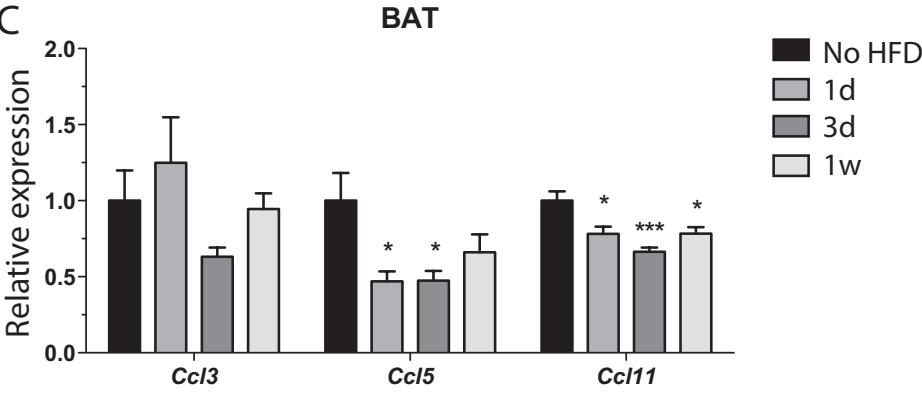

D

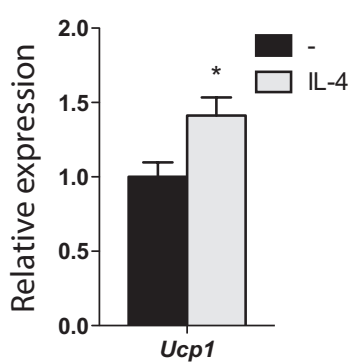

E

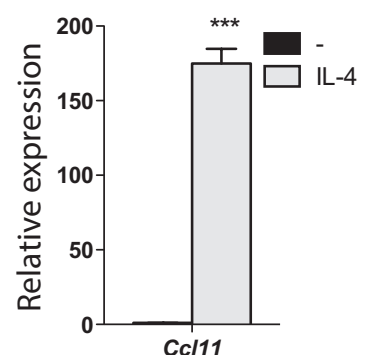

F

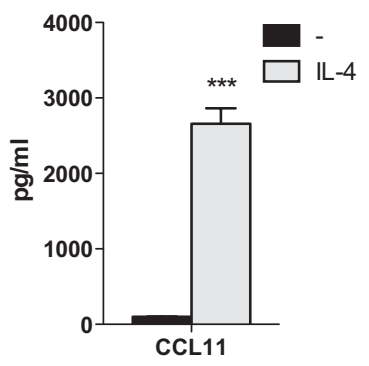

Figure 2

High-fat diet feeding decreases gene expression of eosinophil chemoattractants in adipose tissues. (A) Gene expression of the chemoattractant $\mathrm{CCl}$, $\mathrm{Ccl} / 5$ and $\mathrm{Cc} / 11$. after 1 day, 3 days or 1 week of HFD in EpAT, (B) in ScAT and (C) in BAT. D. Ucp1 gene expression in a brown adipocyte cell line (T37i) that was stimulated with the cytokine IL-4 for $24 \mathrm{~h}$. E. Gene expression and (F) the presence of CCL11 in the supernatant of brown adipocytes as assessed by ELISA after IL-4 stimulation. $n=11$, $* P<0.05, * * P<0.01, * * * P<0.001$ (A, B and C:

1 -way ANOVA with Tukey post-test analysis and the statistical change per group expressed relative to the No HFD group. D, E and F: Student's $t$ test). beiging potential of WAT by decreased attraction of IL-4producing eosinophils.

\section{Adipose tissue depot-specific effects of helminth antigen-induced type 2 immune responses}

Next, we aimed to counteract the HFD-induced decrease in adipose tissue eosinophils in mice by the administration of the helminth antigens SEA and TsSP, both of which are known to induce a type 2 immune response. To this end, we fed $\mathrm{C} 57 \mathrm{Bl} / 6$ mice $(n=12)$ a HFD for 1 week while being treated with the helminth antigens SEA and TsSP (Fig. 3A). These treatments did not cause changes in body weight or BAT, EpAT and ScAT weight (data not shown). Flow cytometry of the adipose tissues revealed that SEA and TsSP caused major effects on immune cell composition of the EpAT (Fig. 3B). SEA and TsSP increased total leukocyte numbers (CD45+ cells) by 6.4 -fold and 4.0 -fold, respectively. This increase in leukocytes was mainly due to a massive accumulation

Published by Bioscientifica Ltd 
A
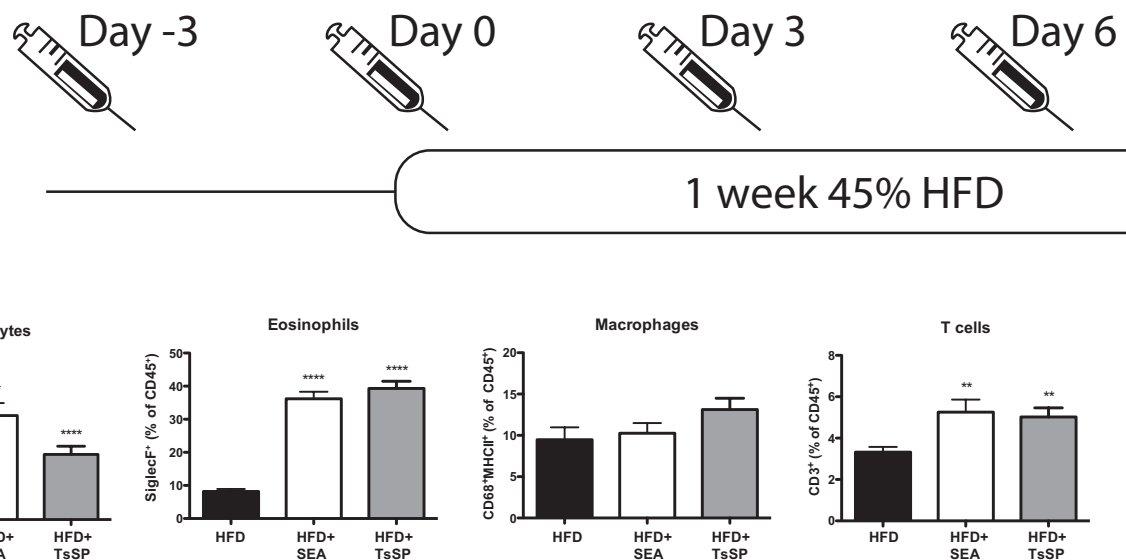

1 week 45\% HFD

\section{В EpAT}
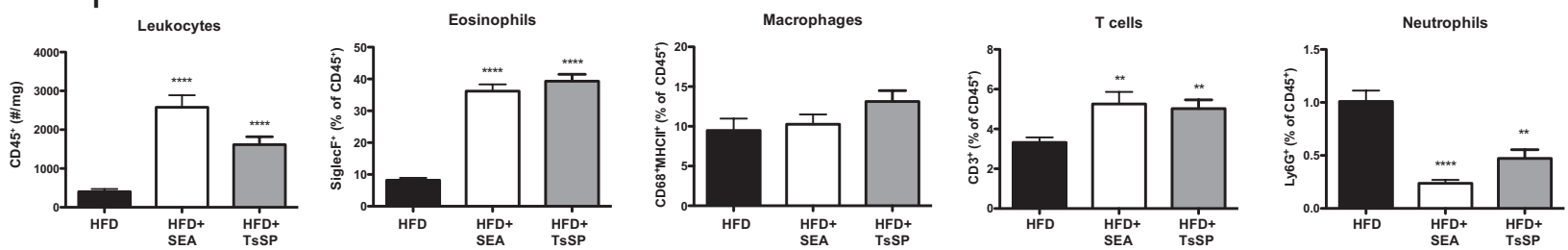

\section{SCAT}

Leukocytes
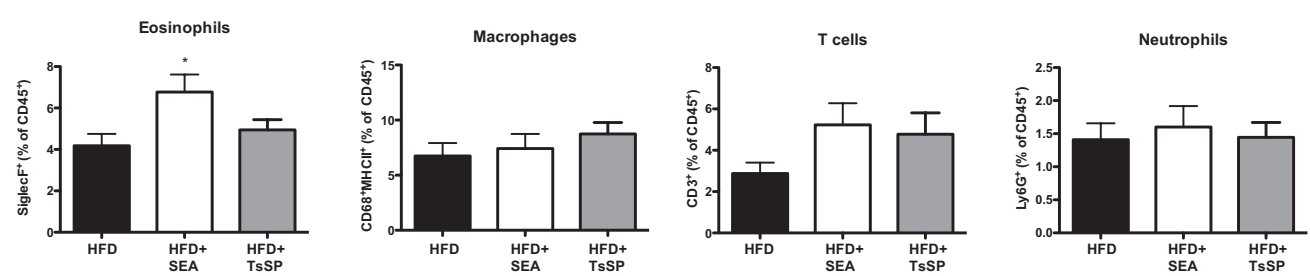

\section{BAT}
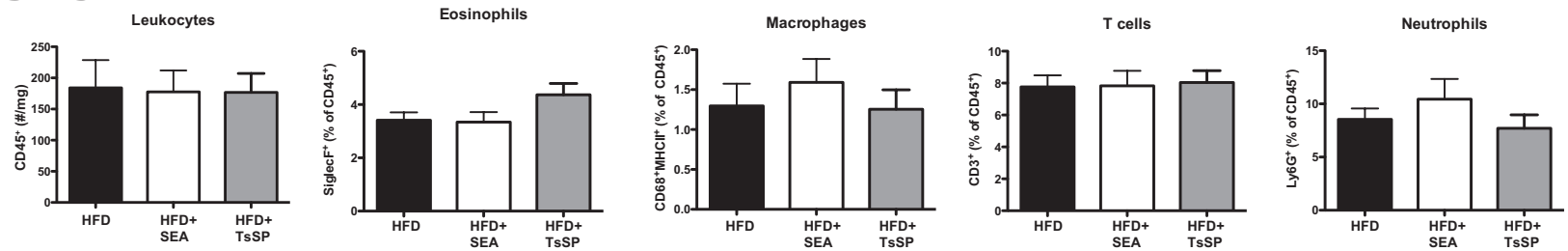

E

EрAT
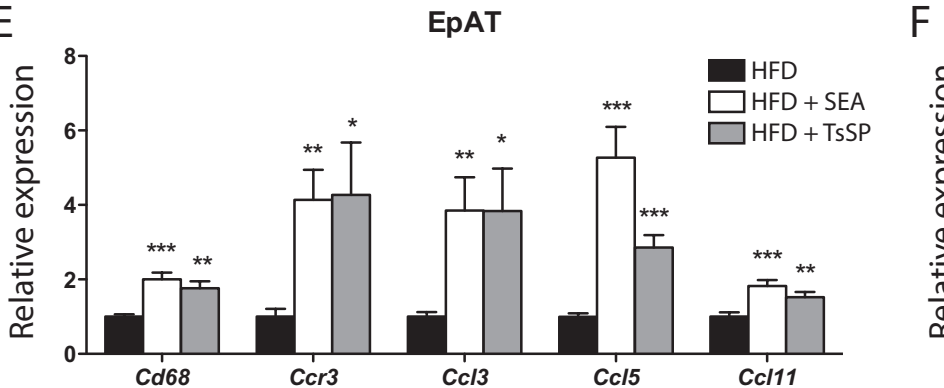

$F$
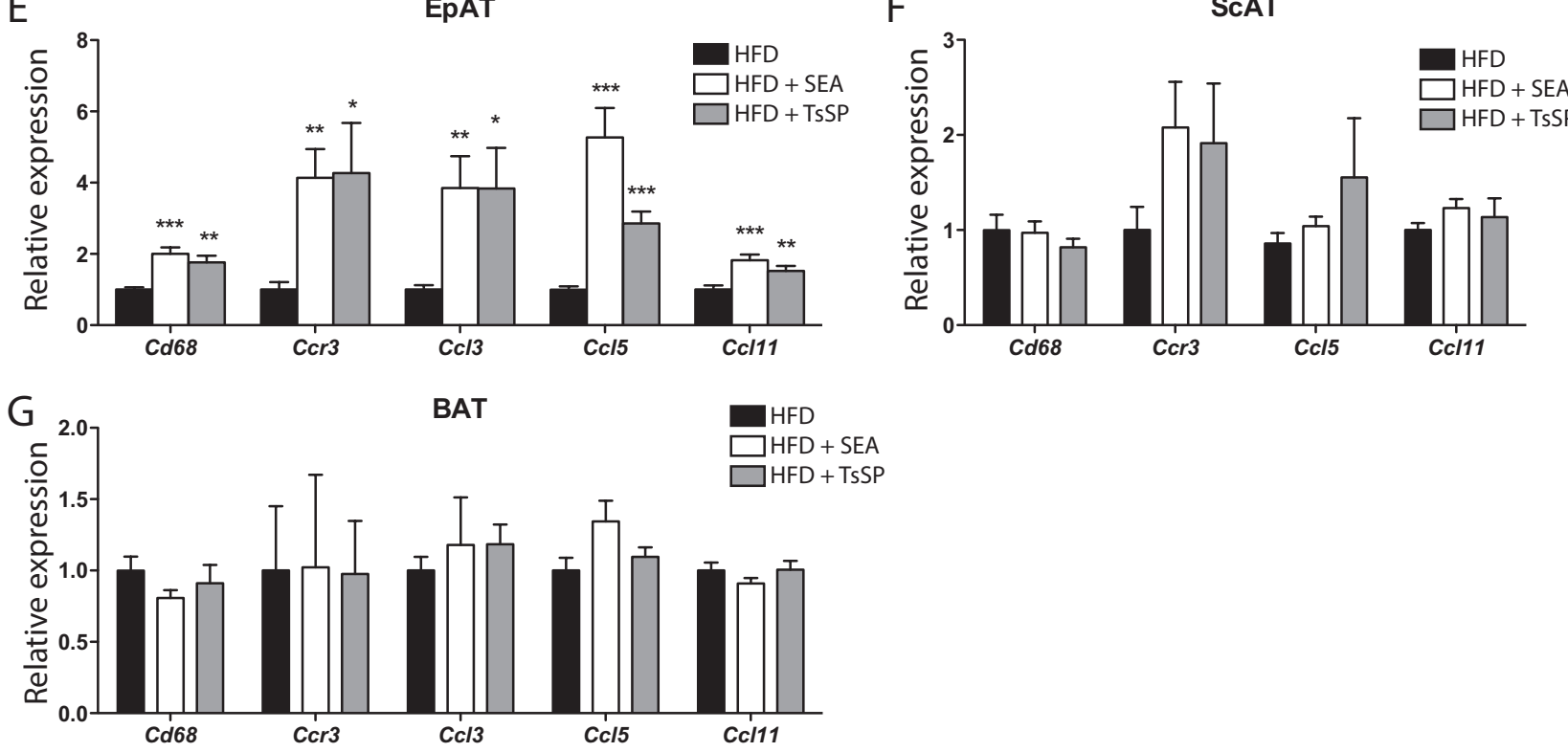

Figure 3

Helminth antigens induce an adipose depot-specific type 2 immune response. (A) Schematic study design. (B) Flow cytometry analysis of total leukocyte number $\left(\mathrm{CD}^{2} 5^{+}\right)$and the \% of eosinophils (SiglecF+), macrophages (CD68+MHCII+), T cells (CD3+) and neutrophils (Ly6G+) within the total leukocyte population of 1-week HFD-fed mice after injections with SEA and TsSP in the stromal vascular fraction of EpAT, (C) SCAT and (D) BAT. (E) Gene expression analysis of macrophage marker $\mathrm{Cd} 68$, eosinophil marker $\mathrm{Ccr} 3$ and chemokines $\mathrm{Ccl}$, $\mathrm{Ccl} / 5$ and $\mathrm{Cc} / 11$ in EpAT (F) SCAT and (G) BAT of SEA and TsSP-treated HFD-fed mice. $n=12, * P<0.05, * * P<0.01, * * * P<0.001$ (Student's $t$ test between the control group and either SEA or TsSP).

\begin{tabular}{|c|c|c|}
\hline $\begin{array}{l}\text { http://jme.endocrinology-journals.org } \\
\text { DOI: 10.1530/JME-17-0112 }\end{array}$ & $\begin{array}{r}\text { C } 2017 \text { Society for Endocrinology } \\
\text { Printed in Great Britain }\end{array}$ & Published by Bioscientifica L \\
\hline
\end{tabular}


of EpAT eosinophils (SiglecF+ cells) after SEA and TsSP treatment. The relative increase in macrophages was not significant in EpAT of the SEA and TsSP groups, although the absolute number of macrophages $\left(\mathrm{CD}^{+}{ }^{+} \mathrm{MHCII}^{+}\right.$ cells) was increased 5.8-fold and 5.2-fold, respectively. Furthermore, SEA treatment increased the percentage of $\mathrm{T}$ cells (CD3+ cells) 1.6-fold and TsSP treatment 1.5fold in EpAT. The relative number of neutrophils $\left(\mathrm{Ly}_{6 \mathrm{G}}{ }^{+}\right.$ cells) decreased, although absolute neutrophil counts were unaffected by the treatments. The effects of the helminth antigens on ScAT were minor compared to
EpAT. We observed a relative increase in eosinophils (+1.6-fold difference) by SEA in ScAT (Fig. 3C). However, we found no changes in immune cell composition of BAT (Fig. 3D). Gene expression analysis confirmed increased macrophage and eosinophil abundance in EpAT upon SEA and TsSP treatment evidenced by increased Cd68 and Ccr3 expression (Fig. 3E). Furthermore, the chemokines responsible for attracting eosinophils Ccl3, Ccl5 and Ccl11 were upregulated in EpAT (Fig. 3E). However, in ScAT and BAT, no significant alterations in gene expression were observed (Fig. 3F and G).

\section{A ScAT}

$\mathrm{B}$
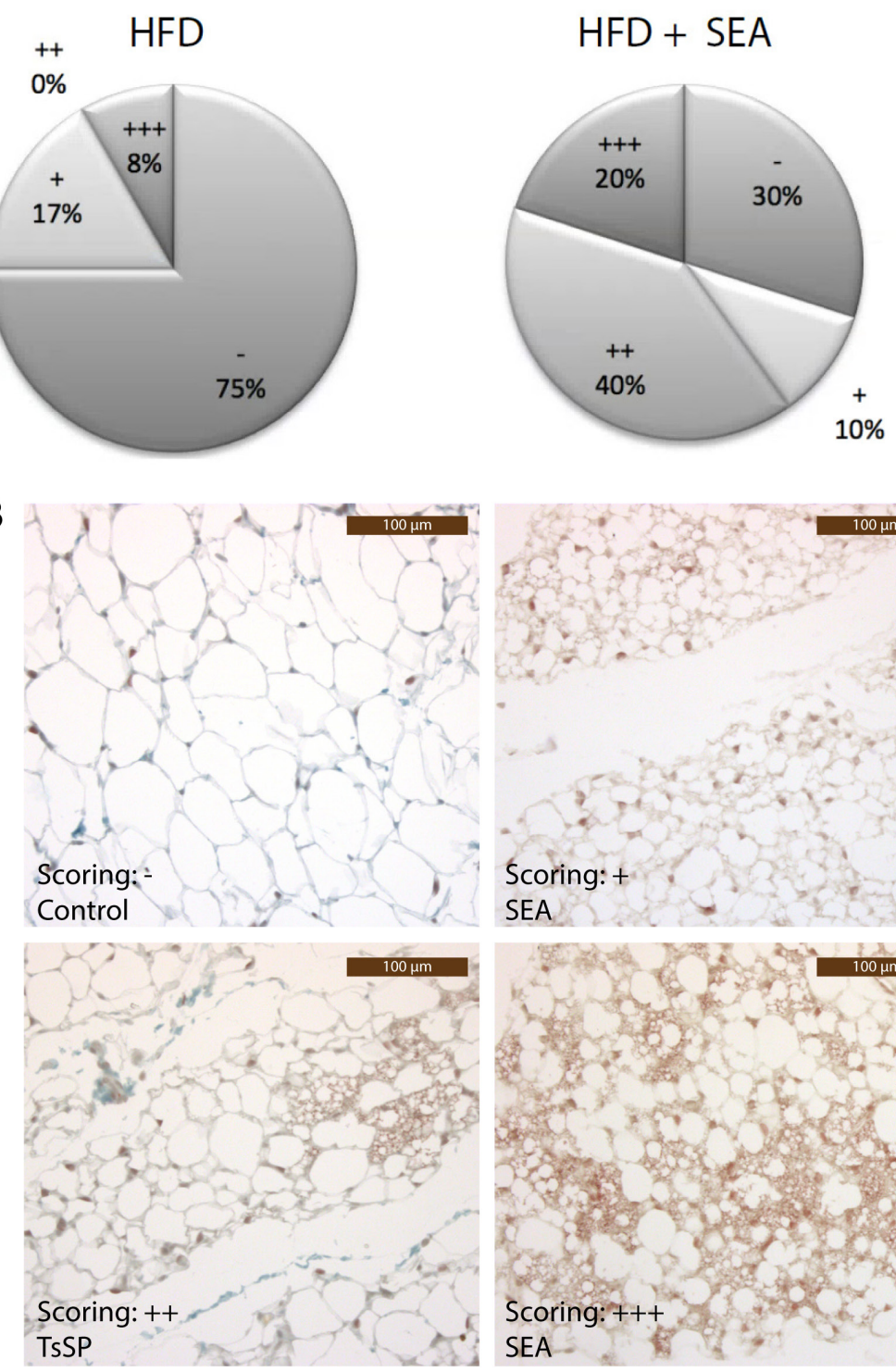
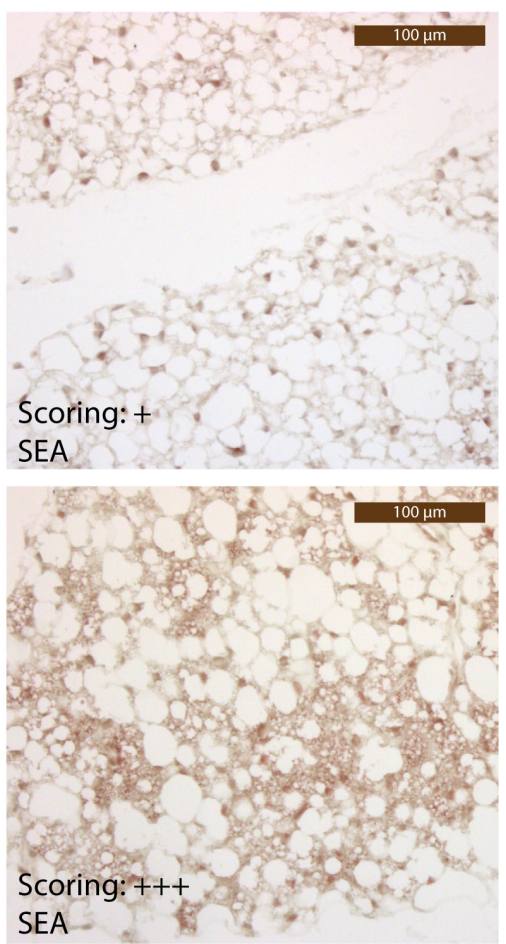

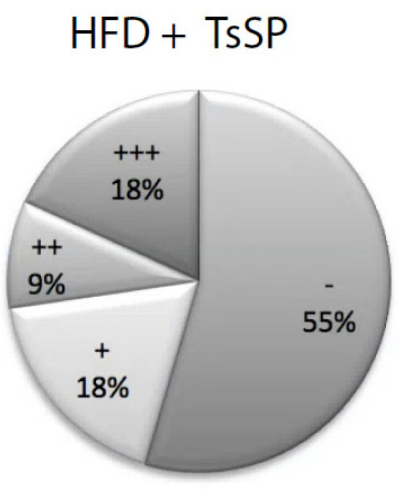

C BAT

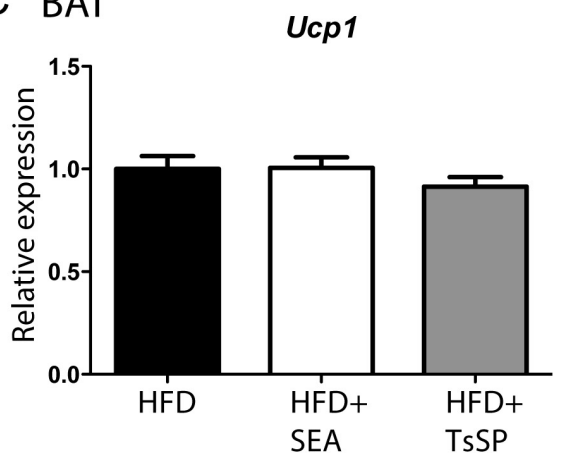

Figure 4

Helminth antigens do not enhance thermogenic capacity of white adipose tissue. (A) Scoring of presence of beige adipocytes in ScAT. Beige adipocytes were defined by a multilocular appearance and positive for UCP1. When present, the quantity was expressed as: +, a few cells; ++; a few areas; and +++, when the section was full of beige adipocytes. (B) Examples of the scoring method of the UCP1 staining. The pictures show examples from the 3 different groups. (C) Gene expression of Ucp1 in BAT after SEA or TsSP treatment in 1-week HFD-fed mice. $n=10-12$.

http://jme.endocrinology-journals.org DOI: 10.1530/JME-17-0112
(C) 2017 Society for Endocrinology Printed in Great Britain
Published by Bioscientifica Ltd 


\section{Helminth antigen treatment does not cause beiging in WAT}

As ScAT is prone to beige adipogenesis, we studied whether the change in leukocyte composition upon helminth antigen treatment in ScAT altered the presence of beige adipocytes. We performed immunohistochemistry for UCP1 on ScAT and scored the occurrence of beiging by UCP1-positive areas and a multilocular appearance in a blinded fashion. This quantification showed that beige adipocytes are slightly more present in ScAT of mice treated with the helminth antigens SEA, but not in mice treated with TsSP (Fig. 4A and B and Table 1). In EpAT, we did not observe any beige adipocytes of either treatment group and thus the major increase in eosinophils in EpAT did not result in beiging in this adipose tissue depot (data not shown). In BAT, Ucp1 expression was unaffected by either SEA or TsSP treatment (Fig. 4C).

\section{Discussion}

Immune cells contribute to the activation of BAT and beiging of WAT, via IL-4-producing eosinophils that stimulate anti-inflammatory macrophages to secrete noradrenaline (Nguyen et al. 2011, Wu et al. 2011, van den Berg et al. 2017). In the current study, we show that short-term HFD causes a rapid decline in eosinophils in EpAT, ScAT and BAT, along with a decrease in chemotactic signals for eosinophils. We also identify a direct crosstalk between eosinophils and brown adipocytes by showing that the cytokine IL- 4 can directly increase Ucp1 gene expression in brown adipocytes in vitro and that brown adipocytes themselves are very much capable of producing and secreting CCL11, a chemokine that recruits eosinophils. Altogether, these findings indicate that a type 2 and anti-inflammatory environment in adipose tissues and the associated beige/ brown adipocyte activation could beneficially alter metabolism. Therefore, we administered helminth antigens

Table 1 Presence of beige adipocytes in ScAT after helminth treatment.

\begin{tabular}{|c|c|c|c|c|c|}
\hline & - & + & ++ & +++ & $P$ value \\
\hline HFD & 9 & 2 & 0 & 1 & \\
\hline HFD + SEA & 3 & 1 & 4 & 2 & 0.06 \\
\hline HFD + TsSP & 6 & 2 & 1 & 2 & 0.59 \\
\hline
\end{tabular}

Values represent the number of mice within the groups that were scored on no beige adipocytes (-), a few beige adipocytes $(+)$, a few beige areas $(++)$ or many beige areas $(+++)$ in SCAT. $P$ value as determined by a chi-square test. $n=10-12$.

SEA, Schistosoma mansoni-soluble egg antigens; TsSP, soluble products of the whipworm Trichuris suis. from SEA and TsSP to 1-week HFD-fed mice to induce a type 2 immune response. We observed very depot-specific effects of SEA and TsSP in which EpAT was highly infiltrated with eosinophils, macrophages and T cells, ScAT also contained higher numbers of eosinophils, whereas no alterations were found in BAT. Furthermore, only SEA treatment showed a slight increase in beige adipogenesis in ScAT.

Eosinophil numbers in tissues depend on signals that influence recruitment, survival and/or cell death. Our data suggest that HFD feeding reduces recruitment of eosinophils into white and brown adipose tissues, as we observe a decrease in expression of the chemotactic signal Ccl3 in EpAT and ScAT, and Ccl5 and Ccl11 in BAT. As these chemokines can be produced by a variety of cell types, including adipocytes and macrophages (Zeyda et al. 2010, Suzuki et al. 2017), these differences in expression are probably due to differences between white and brown adipocytes as well as differences in the immune cell composition within the tissues. Furthermore, 1 week of HFD increased the expression of Ccl11 in EpAT. This increase could be explained by the increase in macrophages that we observe only EpAT, as macrophages are capable of producing CCL11 (Zeyda et al. 2010). As upregulated expression of Ccl11 is apparently not sufficient to restore eosinophil numbers, other factors may be dominant over the chemoattractive effect of CCL11. Eosinophils greatly depend on survival signals, including IL-5, IL-3 and granulocyte macrophage colony-stimulating factor (GM-CSF) (Park \& Bochner 2010). In lean adipose tissue, eosinophils have a slow turnover (10\% in 3 days) (Molofsky et al. 2013), suggesting that lean adipose tissue contains high survival signals. Factors related to obesity and potentially involved in reducing eosinophil numbers include the pro-inflammatory cytokines TNF, which can induce eosinophil apoptosis (Park \& Bochner 2010) and IFN $\gamma$, which can inhibit differentiation and migration of eosinophils (Ochiai et al. 1995). Eosinophil degranulation can also result in cell death (Rosenberg et al. 2013). A rapid decline in eosinophils can be the result of their short half-life, a withdrawal of survival signals or due to an increase in apoptotic factors combined with a decrease in chemoattractants. To unravel a genuine mechanism behind the observed fast drop in adipose tissue eosinophils upon feeding a HFD, future studies should address which signals cause chemokines to decrease, determine whether IL-5 and ILC2s are affected by short-term HFD and search for correlations between apoptotic factors and eosinophil numbers in adipose tissues.

Our results show a marked drop in adipose tissue eosinophils in three distinct adipose tissue depots

Published by Bioscientifica Ltd 
including BAT. We further state that eosinophils immediately decline shortly after the start of a HFD and that this decline is also evident in BAT. Surprisingly, macrophages increased only in EpAT after short-term HFD feeding. Others also report variability in metabolic as well as inflammatory profile between adipose tissue depots (van Beek et al. 2015). Preadipocyte proliferation and recruitment is lower in EpAT than that in ScAT (Lee et al. 2013), and the secretion of inflammatory cytokines are also increased in EpAT relative to ScAT in obese mice (Surmi \& Hasty 2008). Our data suggest that 3 days of HFD is sufficient to induce stress on the existing adipocytes, leading to a rapid recruitment of macrophages. This stress response is possibly caused by the limited capacity for hyperplasia and rapid hypertrophy, which leads to hypoxic conditions and the secretion of signals that attract macrophages. Furthermore, the immune response to HFD might be affected by the age of the mice in the experiment. The next important step would be to confirm such immunological changes in human adipose tissue, as it would be highly relevant if only 1 day of high-fat meals in humans would indeed physiologically resemble 1 day of HFD in mice leading to a dramatic change in adipose tissue immune cell composition. Another important question would be how reversible these effects are.

In the current study, 10 days of treatment with SEA and TsSP induced a massive immune response in EpAT. Others have shown that 4 weeks of SEA treatment and the associated increase in type 2 immunity can be protective in the pathogenesis of metabolic disorders (Hussaarts et al. 2015). We hypothesized that these metabolic improvements were related to BAT activity and beiging of WAT. In our study, the type 2 immune response induced by helminth antigens from both SEA as well as TsSP was very depot specific and was only full blown present in EpAT. Surprisingly, an extreme increase in EpAT eosinophils (34-fold by SEA and 23-fold by TsSP) did not cause beige adipogenesis. This might be explained by a low beiging capacity of EpAT (Waldén et al. 2011). Beiging predominantly occurs in ScAT, likely due to differences in a precursor population for beige adipocytes (SanchezGurmaches \& Guertin 2014). The IL-4R $\alpha$ is highly expressed in adipocyte precursor cells compared to mature adipocytes (Lee et al. 2015), and signalling through this receptor is involved in beige adipogenesis. A different beiging potential between EpAT and ScAT could therefore include differential expression of the IL-4R $\alpha$. Furthermore, in ScAT, SEA treatment increased eosinophil numbers by 1.6-fold, which could be responsible for the trend towards a higher presence of beige adipocytes in ScAT.
In spite of the massive influx of type 2 immune cells upon SEA and TsSP treatment in our study, we did not observe effects on body weight, adipose tissue weight or alterations in beige adipocyte numbers. Our data do not support our hypothesis that the metabolic beneficial phenotype upon treatment with helminth antigens is due to the activation of BAT or beiging of WAT. To unravel possible underlying mechanisms that cause the protective effect of helminths against metabolic disorders in dietinduced obesity, future experiments could explore the effects of helminths on other metabolic organs such as the liver. Furthermore, helminth antigens might directly interfere with insulin signalling pathways, interacting with both immunological as well as metabolic pathways. On the contrary, our short-term study might not have been sufficiently long to observe metabolic effects of BAT activation and beiging. Although administration of IL-4 for 10 days in mice induces beiging of ScAT (Qiu et al. 2014), indicating that this mechanism could take place within this time frame. Furthermore, the mice in this study were housed at $21^{\circ} \mathrm{C}$ and were therefore thermally stressed. This could result in undetectable metabolic changes and a lack of beiging. Studies exploring potential browning agents should therefore be carried out at thermoneutrality $\left(30^{\circ} \mathrm{C}\right.$ in mice) (Cannon \& Nedergaard 2011).

A harmonic co-existence between helminths and their hosts does not often result in severe clinical symptoms. However, as helminths do live of their host, an infection can cause nutritional and vitamin deficiencies, anaemia, growth delays, increased intestinal mobility and increase the risk of other infectious diseases (Guigas \& Molofsky 2015). A clinical application of helminths would be possible if we either find a helminth that does not cause side effects or identify the immunomodulatory components. The helminth Trichuris suis is presumably transient, self-limiting and does not cause adverse effects in healthy subjects (Leonardi et al. 2015). Small scale human trials in allergy, multiple sclerosis and inflammatory bowel disease are already performed with Trichuris suis and shown to improve remission (Leonardi et al. 2015). Using the SEA from Schistosoma mansoni and soluble products of Trichuris suis already circumvents the use of a live parasitic worm infection. Helminths express a wide variety of products, including lipids and proteinand lipid linked glycans, which are present on the surface or secreted extracellularly (van Die \& Cummings 2010). We observed similar overall effects of SEA and TsSP on immune cell composition in adipose tissues, indicating that they stimulate similar pathways that exert the effects in adipose tissue. The molecular mechanisms responsible

Published by Bioscientifica Ltd 
for these type 2 responses, however, appear to differ between these helminths. The major component of TsSP (and secretory products of T. suis) that drives type 2 responses appears to be the lipid mediator prostaglandin E2 (PGE2), which is present in high concentrations in TsSP and is a known type 2 response inducer (van der Pouw Kraan et al. 1995, Kaliński et al. 1997, Laan et al. 2017). In SEA, the RNase Omega1 has been implicated in SEA-induced type 2 responses both in vitro and in vivo (Kaliński et al. 1997, Everts et al. 2009). Before helminth antigens, or mimics thereof, could be therapeutically applied, it will be needed to identify which parasitic signals collectively cause the metabolically beneficial type 2 immune response. Possibly, multiple cooperating signals are required to induce significant physiological effects, such as provided by helminth infection.

In addition to a skewing to type 2 immunity, helminth infections also induce a tolerant phenotype with increased levels of IL-10 and induction of T regs (Maizels $\&$ McSorley 2016). Aside from immunological effects, SEA treatment also has cholesterol-lowering effects, although the atheroprotective effect of SEA was independent from cholesterol lowering in hypercholesterolemic LDLR-/mice (Wolfs et al. 2014).

In conclusion, our data show that HFD disturbs a homeostatic type 2 immunological circuitry in adipose tissue, which cannot be completely reversed by a helminth-induced type 2 response in brown and beigeprone tissues. We also show that IL- 4 can directly activate brown adipocytes in addition to its capacity to polarize macrophages. The crosstalk between immune cells and adipocytes and its role in the regulation of tissue homeostasis is more complex than previously thought with many questions that remain.

\section{Declaration of interest}

The authors declare that there is no conflict of interest that could be perceived as prejudicing the impartiality of the research reported.

\section{Funding}

This work was supported by the Rembrandt Institute of Cardiovascular Science ( $P$ R, M W, E L) and the Netherlands CardioVascular Research Initiative: 'the Dutch Heart Foundation, Dutch Federation of University Medical Centres, the Netherlands Organisation for Health Research and Development and the Royal Netherlands Academy of Sciences' for the GENIUS project 'Generating the best evidence-based pharmaceutical targets for atherosclerosis' (CVON201119). This work was supported by the Netherlands Heart Foundation (junior postdoc grant to JVdB), the Netherlands Organization for Scientific Research (NWO) (VENI to JVdB, VICl grant to E L), the Dutch Diabetes Foundation (Junior fellowship 2015.81.1808 to M B) and the European Research Council (ERC con grant to $\mathrm{E} \mathrm{L}$ ).

\section{Acknowledgements}

The authors are grateful for using the T37i brown adipocyte cell line provided by Marc Lombès (Institut National de la Santé et de la Recherche Médicale, Le Kremlin-Bicêtre, France) and the use of Schistosoma mansoniSEA, which was kindly provided by Fred Lewis (Biomedical Research Institute, Rockville, MD, USA).

\section{References}

Berbée JFP, Boon MR, Khedoe PPSJ, Bartelt A, Schlein C, Worthmann A Kooijman S, Hoeke G, Mol IM, John C, et al. 2015 Brown fat activation reduces hypercholesterolaemia and protects from atherosclerosis development. Nature Communications $\mathbf{6}$ article 6356. (doi:10.1038/ncomms7356)

Boros DL \& Warren KS 1970 Delayed hypersensitivity-type granuloma formation and dermal reaction induced and elicited by a soluble factor isolated from Schistosoma mansoni eggs. Journal of Experimental Medicine 132 488. (doi:10.1084/jem.132.3.488)

Cannon B \& Nedergaard J 2004 Brown adipose tissue: function and physiological significance. Physiological Reviews $\mathbf{8 4}$ 277-359. (doi:10.1152/physrev.00015.2003)

Cannon B \& Nedergaard J 2011 Nonshivering thermogenesis and its adequate measurement in metabolic studies. Journal of Experimental Biology 214 242-253. (doi:10.1242/jeb.050989)

Everts B, Perona-Wright G, Smits $\mathrm{HH}$, Hokke $\mathrm{CH}$, van der Ham AJ, Fitzsimmons CM, Doenhoff MJ, van der Bosch J, Mohrs K, Haas H, et al. 2009 Omega-1, a glycoprotein secreted by Schistosoma mansoni eggs, drives Th2 responses. Journal of Experimental Medicine 206 1673. (doi:10.1084/jem.20082460)

Guigas B \& Molofsky AB 2015 A worm of one's own: how helminths modulate host adipose tissue function and metabolism. Trends in Parasitology 31 435-441. (doi:10.1016/j.pt.2015.04.008)

Hoeksema M, Laan L, Postma J, Cummings R, de Winther M, Dijkstra C, van Die I \& Kooij G 2016 Treatment with Trichuris suis soluble products during monocyte-to-macrophage differentiation reduces inflammatory responses through epigenetic remodeling. FASEB Journal 30 2826-2836. (doi:10.1096/fj.201600343R)

Hussaarts L, García-Tardón N, van Beek L, Heemskerk MM, Haeberlein S, van der Zon GC, Ozir-Fazalalikhan A, Berbée JFP, Willems van Dijk $\mathrm{K}$, van Harmelen V, et al. 2015 Chronic helminth infection and helminth-derived egg antigens promote adipose tissue M2 macrophages and improve insulin sensitivity in obese mice. FASEB Journal 29 3027-3039. (doi:10.1096/fj.14-266239)

Kaliński P, Hilkens C, Snijders A, Snijdewint F \& Kapsenberg M 1997 Dendritic cells, obtained from peripheral blood precursors in the presence of PGE2, promote Th2 responses. Advances in Experimental Medicine and Biology 417 363-367. (doi:10.1007/978-1-4757-9966-8_59)

Klaver EJ, Kuijk LM, Laan LC, Kringel H, van Vliet SJ, Bouma G, Cummings RD, Kraal G \& van Die I 2013 Trichuris suis-induced modulation of human dendritic cell function is glycan-mediated. International Journal for Parasitology 43 191-200. (doi:10.1016/j.ijpara.2012.10.021)

Kuijk LM, Klaver EJ, Kooij G, Pol SM, Heijnen P \& Bruijns SC 2012 Soluble helminth products suppress clinical signs in murine experimental autoimmune encephalomyelitis and differentially modulate human dendritic cell activation. Molecular Immunology $\mathbf{5 1}$ 210-218. (doi:10.1016/j.molimm.2012.03.020)

Laan LC, Williams AR, Stavenhagen K, Giera M, Kooij G, Vlasakov I, Kalay H, Kringel H, Nejsum P, Thamsborg SM, et al. 2017 The whipworm (Trichuris suis) secretes prostaglandin E2 to suppress proinflammatory properties in human dendritic cells. FASEB Journal 31 719-731. (doi:10.1096/fj.201600841R)

Lee M-J, Wu Y \& Fried SK 2013 Adipose tissue heterogeneity: implication of depot differences in adipose tissue for obesity complications. Molecular Aspects of Medicine 34 1-11. (doi:10.1016/j.mam.2012.10.001)

Published by Bioscientifica Ltd. 
Lee M-W, Odegaard JI, Mukundan L, Qiu Y, Molofsky AB, Nussbaum JC, Yun K, Locksley RM \& Chawla A 2015 Activated type 2 innate lymphoid cells regulate beige fat biogenesis. Cell 160 74-87. (doi:10.1016/ j.cell.2014.12.011)

Leonardi I, Frey I \& Rogler G 2015 Helminth therapy for organic diseases? Translational Research 166 586-601. (doi:10.1016/ j.trsl.2015.06.016)

Lumeng CN, Bodzin JL \& Saltiel AR 2007 Obesity induces a phenotypic switch in adipose tissue macrophage polarization. Journal of Clinical Investigation 117 175-184. (doi:10.1172/JCI29881)

Maizels RM \& McSorley HJ 2016 Regulation of the host immune system by helminth parasites. Journal of Allergy and Clinical Immunology $\mathbf{1 3 8}$ 666-675. (doi:10.1016/j.jaci.2016.07.007)

Molofsky AB, Nussbaum JC, Liang H-E, Van Dyken SJ, Cheng LE, Mohapatra A, Chawla A \& Locksley RM 2013 Innate lymphoid type 2 cells sustain visceral adipose tissue eosinophils and alternatively activated macrophages. Journal of Experimental Medicine 210 535. (doi:10.1084/jem.20121964)

Nguyen KD, Qiu Y, Cui X, Goh YPS, Mwangi J, David T, Mukundan L, Brombacher F, Locksley RM \& Chawla A 2011 Alternatively activated macrophages produce catecholamines to sustain adaptive thermogenesis. Nature 480 104-108. (doi:10.1038/nature10653)

Ochiai K, Iwamoto I, Takahashi H, Yoshida S, Tomioka H \& Yoshida S 1995 Effect of IL-4 and interferon-gamma (IFN-gamma) on IL-3- and IL-5-induced eosinophil differentiation from human cord blood mononuclear cells. Clinical and Experimental Immunology 99 124-128. (doi:10.1111/j.1365-2249.1995.tb03482.x)

Park YM \& Bochner BS 2010 Eosinophil survival and apoptosis in health and disease. Allergy, Asthma and Immunology Research 2 87-101.

Qiu Y, Nguyen Khoa D, Odegaard Justin I, Cui X, Tian X, Locksley Richard M, Palmiter Richard D \& Chawla A 2014 Eosinophils and type 2 cytokine signaling in macrophages orchestrate development of functional beige fat. Cell $\mathbf{1 5 7}$ 1292-1308. (doi:10.1016/ j.cell.2014.03.066)

Roberts-Toler C, O'Neill BT \& Cypess AM 2015 Diet-induced obesity causes insulin resistance in mouse brown adipose tissue. Obesity $\mathbf{2 3}$ 1765-1770. (doi:10.1002/oby.21134)

Rosenberg HF, Dyer KD \& Foster PS 2013 Eosinophils: changing perspectives in health and disease. Nature Reviews Immunology $\mathbf{1 3}$ 9-22. (doi:10.1038/nri3341)

Saito M, Okamatsu-Ogura Y, Matsushita M, Watanabe K, Yoneshiro T, Nio-Kobayashi J, Iwanaga T, Miyagawa M, Kameya T, Nakada K, et al. 2009 High incidence of metabolically active brown adipose tissue in healthy adult humans: effects of cold exposure and adiposity. Diabetes 58 1526-1531. (doi:10.2337/db09-0530)

Sanchez-Gurmaches J \& Guertin DA 2014 Adipocytes arise from multiple lineages that are heterogeneously and dynamically distributed. Nature Communications 5 article 4099. (doi:10.1038/ncomms5099)

Sanchez-Gurmaches J, Hung C-M \& Guertin DA 2016 Emerging complexities in adipocyte origins and identity. Trends in Cell Biology 26 313-326. (doi:10.1016/j.tcb.2016.01.004)

Shabalina IG, Petrovic N, de Jong Jasper MA, Kalinovich Anastasia V, Cannon B \& Nedergaard J 2013 UCP1 in brite/beige adipose tissue mitochondria is functionally thermogenic. Cell Reports $\mathbf{5}$ 1196-1203. (doi:10.1016/j.celrep.2013.10.044)

Surmi BK \& Hasty AH 2008 Macrophage infiltration into adipose tissue: initiation, propagation and remodeling. Future Lipidology 3 545-556. (doi:10.2217/17460875.3.5.545)

Suzuki T, Gao J, Ishigaki Y, Kondo K, Sawada S, Izumi T, Uno K, Kaneko K, Tsukita S, Takahashi K, et al. 2017 ER stress protein CHOP mediates insulin resistance by modulating adipose tissue macrophage polarity. Cell Reports 18 2045-2057. (doi:10.1016/j.celrep.2017.01.076)

van Beek L, van Klinken JB, Pronk ACM, van Dam AD, Dirven E, Rensen PCN, Koning F, Willems van Dijk K \& van Harmelen V 2015 The limited storage capacity of gonadal adipose tissue directs the development of metabolic disorders in male C57Bl/6J mice. Diabetologia 58 1601-1609. (doi:10.1007/s00125-015-3594-8)

van den Berg SM, van Dam AD, Rensen PCN, de Winther MPJ \& Lutgens E 2017 Immune modulation of brown(ing) adipose tissue in obesity. Endocrine Reviews 38 46-68.

van der Lans AAJJ, Hoeks J, Brans B, Vijgen GHEJ, Visser MGW, Vosselman MJ, Hansen J, Jorgensen JA, Wu J, Mottaghy FM, et al. 2013 Cold acclimation recruits human brown fat and increases nonshivering thermogenesis. Journal of Clinical Investigation $\mathbf{1 2 3}$ 3395-3403. (doi:10.1172/JCI68993)

van der Pouw Kraan TC, Boeije LC, Smeenk RJ, Wijdenes J \& Aarden LA 1995 Prostaglandin-E2 is a potent inhibitor of human interleukin 12 production. Journal of Experimental Medicine $\mathbf{1 8 1}$ 775. (doi:10.1084/jem.181.2.775)

van Die I \& Cummings RD 2010 Glycan gimmickry by parasitic helminths: a strategy for modulating the host immune response? Glycobiology 20 2-12. (doi:10.1093/glycob/cwp140)

van Marken Lichtenbelt WD, Vanhommerig JW, Smulders NM, Drossaerts JMAFL, Kemerink GJ, Bouvy ND, Schrauwen P \& Teule GJJ 2009 Cold-activated brown adipose tissue in healthy men. New England Journal of Medicine 360 1500-1508. (doi:10.1056/NEJMoa0808718)

Waldén TB, Hansen IR, Timmons JA, Cannon B \& Nedergaard J 2011 Recruited vs nonrecruited molecular signatures of brown, 'brite,' and white adipose tissues. American Journal of Physiology - Endocrinology and Metabolism 302 E19.

Weisberg SP, McCann D, Desai M, Rosenbaum M, Leibel RL \& Ferrante AW Jr 2003 Obesity is associated with macrophage accumulation in adipose tissue. Journal of Clinical Investigation $\mathbf{1 1 2}$ 1796-1808. (doi:10.1172/JCI200319246)

Wolfs IMJ, Stöger JL, Goossens P, Pöttgens C, Gijbels MJJ, Wijnands E, van der Vorst EPC, van Gorp P, Beckers L, Engel D, et al. 2014 Reprogramming macrophages to an anti-inflammatory phenotype by helminth antigens reduces murine atherosclerosis. FASEB Journal 28 288-299. (doi:10.1096/fj.13-235911)

Wu D, Molofsky AB, Liang H-E, Ricardo-Gonzalez RR, Jouihan HA, Bando JK, Chawla A \& Locksley RM 2011 Eosinophils sustain adipose alternatively activated macrophages associated with glucose homeostasis. Science 332 243-247. (doi:10.1126/science.1201475)

Yoneshiro T \& Saito M 2015 Activation and recruitment of brown adipose tissue as anti-obesity regimens in humans. Annals of Medicine 47 133-141. (doi:10.3109/07853890.2014.911595)

Yoneshiro T, Aita S, Matsushita M, Kayahara T, Kameya T, Kawai Y, Iwanaga T \& Saito M 2013 Recruited brown adipose tissue as an antiobesity agent in humans. Journal of Clinical Investigation $\mathbf{1 2 3}$ 3404-3408. (doi:10.1172/JCI67803)

Zennaro MC, Le Menuet D, Viengchareun S, Walker F, Ricquier D \& Lombès M 1998 Hibernoma development in transgenic mice identifies brown adipose tissue as a novel target of aldosterone action. Journal of Clinical Investigation $\mathbf{1 0 1}$ 1254-1260. (doi:10.1172/JCI1915)

Zeyda M, Gollinger K, Kriehuber E, Kiefer FW, Neuhofer A \& Stulnig TM 2010 Newly identified adipose tissue macrophage populations in obesity with distinct chemokine and chemokine receptor expression. International Journal of Obesity $\mathbf{3 4}$ 1684-1694. (doi:10.1038/ijo.2010.103)

Received in final form 6 July 2017

Accepted 10 July 2017

Accepted Preprint published online 10 July 2017 http://jme.endocrinology-journals.org

DOI: 10.1530/JME-17-0112
๑) 2017 Society for Endocrinology Printed in Great Britain
Published by Bioscientifica Ltd 